\title{
ILIAC VEIN OBSTRUCTION DUE TO A RETROPERITONEAL ABSCESS
}

\author{
BY \\ P. J. LIPSITZ \\ From the Department of Pediatrics and Contagious Diseases, Cleveland Metropolitan General Hospital, and \\ the Department of Pediatrics, Western Reserve University School of Medicine, Cleveland, Ohio
}

(RECEIVED FOR PUBLICATION OCTOBER 20, 1959)

The occurrence of iliac vein obstruction due to a retroperitoneal abscess in infants is a rare phenomenon. This presentation of a case may be of value in the diagnosis of other cases.

\section{Case Report}

L.M., a newborn white female infant, was admitted on February 8, 1959, to Cleveland Metropolitan General Hospital as a premature home delivery. Her birth weight was $1,840 \mathrm{~g}$. (4 lb.), and expected date of delivery was March 18, 1959. The mother did not have antenatal care. On the second day in hospital the baby was noted to be jaundiced. The mother's blood was group A Rh positive and the baby was group $\mathrm{O} R \mathrm{R}$ positive. On the fourth day in hospital the maximum bilirubin level was $19 \mathrm{mg} . \%$. The baby did not feed well. There was no evidence of septicaemia as indicated by a negative urinalysis and normal spinal fluid. On the tenth day a femoral venipuncture was done for blood cultures and serum chemistries. The side from which the blood was drawn was not recorded. The blood cultures were negative and all the serum chemistry was within the normal range.

At the age of 25 days, swelling of the right thigh and foot was noted. The leg was oedematous and showed some discolouration. Some areas over the thigh were fluctuant but no localizing abscess was present. Inguinal nodes were not palpable. The measurements above the knees were: right $14 \mathrm{~cm}$., left $12 \mathrm{~cm}$. The W.B.C. was $26,850 /$ c.mm. (neutrophils $70 \%$ ). An infection was suspected. Radiograph of the hips revealed no evidence of osteomyelitis of the hip or any intra-articular fluid. The fluctuant area was aspirated but no free fluid was obtained. Material from the needle was cultured and blood cultures were also drawn. The blood cultures were negative but the aspirate grew haemolytic Staphylococcus aureus coagulase positive; resistant to penicillin; sensitive to erythromycin, streptomycin, kanamycin, furadantin, neomycin, chloramphenicol. Nose culture grew Staphylococcus albus. Chloramphenicol treatment was started.

On the 26th day in hospital circumferences of the thighs were: right $14.5 \mathrm{~cm}$.; left $12 \mathrm{~cm}$.; W.B.C. $18,500 \mathrm{c.mm}$. (neutrophils 54\%). The right leg seemed to become more oedematous and on the 30th day measured $15 \mathrm{~cm}$.; left $12 \mathrm{~cm}$; W.B.C. 25,500 c.mm. (neutrophils $83 \%$ ). The diagnosis of thrombophlebitis was entertained at this point. The infant was continued on chloramphenicol and clinically there was no change in the condition of the leg. She held the right leg abducted and externally rotated and resented having the leg moved. No abdominal masses were palpable. Rectal examination was normal. A low grade fever with temperatures of $37^{\circ} \mathrm{C}$. to $38^{\circ} \mathrm{C}$. now appeared.

On the 32nd day an intravenous pyelogram was done, but no pathological process was noticed. A cystogram on the 33rd day showed no evidence of displacement or filling defect of the bladder. Phlebograms were not done. A mass was now palpable in the right lower quadrant deep in the pelvis extending to Poupart's ligament. Rectal examination revealed a bogginess in the area of the right iliopsoas muscle. The diagnosis of retroperitoneal abscess with iliac vein obstruction which had been previously entertained was now confirmed.

On the 34th day a retroperitoneal dissection was done with counterincision of the right thigh and $20 \mathrm{ml}$. of greenish yellow pus was removed. Gram stain revealed gram positive cocci and culture grew haemolytic Staphylococcus aureus coagulase positive; resistant to penicillin, streptomycin, erythromycin, chloramphenicol; sensitive to neomycin, kanamycin, furadantin. Pus was obtained in the operative field by pressure on the thigh and a guide introduced behind the psoas fascia anteriorly extended to the upper and medial aspect of the right thigh. $60 \mathrm{ml}$. of blood was given to the infant. On the second postoperative day measurements of the thighs were: right $12.75 \mathrm{~cm}$., left $11 \mathrm{~cm}$.

The chloramphenicol was discontinued on the 46th day. The infant now weighed $2,660 \mathrm{~g}$. $(5 \mathrm{lb} .14 \mathrm{oz}$.). She was discharged at the age of 2 months, completely well and when seen at the out-patient clinic two weeks later the leg was normal in size compared to the left and the wound had healed. At the age of 6 months the leg and hip were normal. Radiograph of the hip showed no dislocation or destruction of the head of the femur.

\section{Discussion}

The diagnosis of iliac vein obstruction due to a retroperitoneal abscess should be considered when an infant presents with an oedematous leg and dis- 
tended veins. In 1957 Grunwaldt and Tomsovic reported two cases in male infants secondary to circumcision, and since then no further reports have appeared in the literature. In the differential diagnosis, thrombophlebitis, hip disease or, if it follows a femoral vein puncture, an infected haematoma must also be considered. The diagnosis of a retroperitoneal abscess can be made by the palpation of a mass in the lower abdomen and on rectal examination. Special investigations such as intravenous pyelography, cystograms and phlebograms may be confirmatory.

Once the diagnosis has been made surgical drainage is the only method of treatment. The aetiological organism can then be determined and appropriate antibiotic therapy instituted. It was distressing to note that the organisms isolated from this case were Staphylococcus aureus coagulase positive, and that they were resistant to erythromycin and chloramphenicol, the drugs most frequently used for staphylococcal infections.

The pathogenesis of this infection presents many problems for consideration. In this report we must consider the site of femoral vein puncture as the route of introduction of the infection.

Grunwaldt and Tomsovic (1957) report two cases of iliac vein obstruction in male infants both aged 3 weeks. They conclude that the primary site of infection was the circumcision site. Inguinal nodes were palpable in their cases. This suggests that the retroperitoneal infection in those cases was possibly related to skin infection, most likely the penis.

Gross (1953) reports that in some cases of iliac adenitis the portal of entry of the infection may be found on the leg or around the arms, but the initial lesion is often healed by the time the patient presents with abdominal complaints or limp. In most cases the infection skips the femoral and inguinal glands and lodges solely in the iliac group. The age range of the 20 cases reported varies from 1 to 11 years. Sex incidence is not discussed.

Reference is made in the literature to obstructive anomalies of the iliac veins with growth shortening of the ipsilateral leg (Peck, 1957). The obstruction can be either an acquired or developmental disease represented by adhesions in the retroaortic portion of the vein, or pathology due to congenital valves or septa.

Ross (1954) mentions a case of osteomyelitis of the hip with complete destruction of the head and neck of femur following a femoral venipuncture in a premature infant, but concludes that the infection of the hip resulted from a haematogenous spread from a distant focus rather than from a venipuncture in the region of the hip.
Howard (1957) reports five cases of sepsis in normal and premature infants with localization in the hip joint, but does not relate this to femoral venipuncture. He concludes that in one case the probable portal of entry of the infection was from an injection of vitamin K. Three of his five cases had received vitamin $\mathrm{K}$ and other subcutaneous injections.

Neuhof and Arnheim (1944) quote two cases of infected haematomas as a source of infection with formation of iliac retroperitoneal abscess.

Bosch, Kengeter and Beling (1950) discuss femoral venipuncture in 100 adult patients. They list the possible complications, namely, injury to femoral vein, penetration of a viscus in the presence of unrecognized femoral hernia, haematoma, abscess or cellulitis, penetration of psoas abscess, local ecchymosis, thrombophlebitis, phlebothrombosis or arteriovenous fistula.

Sieber and Ferguson (1959) review some of the surgical and orthopaedic aspects of infections in newly-born and young infants. From 1940 to 1957 31 cases, who had had bacterial infections of bones and joints, were found. Nine of these 31 infants had septic hips. They mention femoral vein puncture as an aetiological factor in the localization of infection in the hip joint of four infants.

This report differs from any of the previous cases described in that this occurred in a female infant, no intramuscular or subcutaneous injections were given, and the only probable portal of entry of the infection was the femoral venipuncture.

The jaundice was considered as physiological in a premature infant. In this case there was no evidence of any septicaemia as indicated by the negative blood cultures on the 10th and 25th hospital days. A haematoma at the site of venipuncture or a thrombophlebitis may possibly have been a predisposing factor in the development of this infection. It is virtually impossible to prove that the femoral venipuncture resulted in this retroperitoneal abscess, but it would seem that this is the most likely portal of entry of the infection.

When one asks other paediatricians if they have ever encountered any complication of femoral venipuncture, they may recall some cases of hip infection or osteomyelitis of the femur, but they cannot prove that this was a complication of femoral venipuncture.

In this hospital femoral vein punctures are regarded as hazardous procedures. In the first few days of life the umbilical vein can easily be entered and many paediatricians feel that adequate blood samples can be obtained by this route. It has been disturbing to note the number of positive blood 
cultures obtained before starting exchange transfusions, and an investigation was conducted to determine the feasibility of using this procedure for routine blood cultures in newborns. In a report (Lipsitz and Cornet, unpublished) it will be shown how 15 out of 33 blood cultures obtained from healthy newborns were contaminated with the same organisms as cultured from the umbilical stump. It is strongly recommended that this procedure should not be used.

\section{Summary}

This report is of a case of retroperitoneal abscess with iliac vein obstruction in a premature white female infant aged 25 days. The diagnosis was made on palpation of a mass in the right lower quadrant and rectal examination revealing some bogginess on the right side. It is most likely that the portal of entry was from the site of a femoral vein puncture.

The possible hazards of femoral venipuncture are discussed. Reference is also made to the validity of blood cultures obtained via the umbilical vein.
A review of some of the cases of hip joint pathology in premature infants does not relate the infection to venipuncture, but more likely to subcutaneous and intramuscular injections.

For permission to report this case it is a pleasure to record my thanks to Dr. F. C. Robbins, Director of Pediatrics, and Dr. F. A. Simeone, Director of Surgery. I wish to thank Dr. E. A. Mortimer, Jr., for his helpful criticism and advice.

\section{REFERENCES}

Bosch, D. T., Kengeter, J. P. and Beling, C. A. (1950). Femoral venipuncture Amer, $J$ Surg, $79,722$.

Gross, R. E. (1953). The Surgery of Infancy and Childhood. p. 392. Saunders, Philadelphia and London,

Grunwaldt, E., and Tomsovic, E. J. (1957). Iliac vein obstruction caused by retroperitoneal abscess in newborn infants. J. Paediat., $\mathbf{5 0 , 3 6 1 .}$.

Howard, P. J. (1957). Sepsis in normal and premature infants with localization in the hip joint. Pediatrics, 20, 279.

Neuhof, H. and Arnheim, E. E. (1944). Acute retroperitoneal abscess and phlegmon. Study of 65 cases. Ann. Surg., 119, 741.

Peck, M. E. (1957). Obstructive anomalies of the iliac vein associated with growth shortening in the ipsilateral extremity. Ibid., 146, 619.

Ross, D. W. (1954). Acute suppurative arthritis of the hip in premature infants. J. Amer. med. Ass., $156(4), 303$, Sept.

Sieber, W. K. and Ferguson, A. B. (1959). Surgical and orthopedic aspects of infections in newly born and young infants. Pediatrics, $24,145$. 